\title{
Les réseaux de production collaborative de connaissances
}

Mélanie Dulong de Rosnay

Les réseaux facilitent la coordination de projets et le travail collaboratif en ligne. L'économie de l'information en réseau provoque une transformation des modèles de production des connaissances qui empruntent un modèle de gouvernance horizontale par les communautés elles-mêmes pour créer des ressources partagées, des biens communs (Aigrain, 2005 ; Benkler, 2009; Ostrom, 1990 ; Vecam, 2011). Ces modèles de production sont organisés autour d'une gouvernance décentralisée par les pairs, sans point de contrôle central. Ces pratiques diffèrent des modes traditionnels de production organisés par l'État ou par le marché qui sont basés sur un modèle de gouvernance vertical et propriétaire, dans lesquels l'appropriation de la production est contrôlée par d'autres règles telles que le prix ou l'application de critères extérieurs. La prise de décision décentralisée et collective par des pairs que l'on trouve dans les réseaux de production collaborative de connaissances est facilitée par les réseaux numériques, qui rendent également possible la diffusion des connaissances à un coût marginal nul. La production à un coût très limité est quant à elle rendue possible par la participation volontaire des acteurs des réseaux (Shirky, 2010).

L'analyse des réseaux collaboratifs de production de connaissances demande de faire appel à des théories économiques et politiques qui se construisent à partir de l'observation et de la participation à ces réseaux de production et de distribution de biens par les pairs.

\section{Les réseaux pair-à-pair sans autorisation}

Les productions résultant de cette économie soulèvent des questions juridiques quant à leur statut et à leur appropriation. On trouve tout d'abord la distribution dans ces réseaux de créations non autorisées. Il peut s'agir de l'œuvre originale elle-même en cas de diffusion à l'identique, ou d'adaptations réalisées sans l'accord des titulaires de droits des auvres préexistantes. Cependant, ces sources partagées peuvent s'avérer de meilleure qualité que les productions diffusées commercialement et répondent donc à une carence du marché officiel. On citera en exemple les réseaux de sous-titrage des séries télévisées qui s'auto-organisent à travers des forums et publient les sous-titres plus rapidement que les producteurs ou les diffuseurs commerciaux. Ces adaptations réalisées sans l'autorisation des ayants-droits sont en droit constitutives de contrefaçon. Les réseaux d'échange de fichiers pair-à-pair, dotés d'une architecture technique distribuée présentent pour leur part une meilleure vitesse de téléchargement que les services commerciaux, et proposent des œuvres rares qui ne sont pas disponibles en version numérique sur les plateformes commerciales. De plus, celles-ci placent des verrous techniques qui limitent l'usage et la copie des œuvres, rendant les fichiers distribués par les pairs encore plus attractifs car non-bridés et lisibles sans restriction. Ces réseaux pair-à-pair apportent dès lors une diversité culturelle compatible avec l'économie de la longue traîne : une majorité de titres très connus cohabite avec une minorité foisonnante de titres peu demandés (Bodó, Lakatos, 2012). Là encore, ils répondent à une carence du marché.

\section{Les réseaux de production par les pairs sur la base de biens communs}

Les réseaux de production ont aussi permis l'émergence de créations collectives très structurées, dont les modalités de gouvernance et d'appropriation appartiennent également aux pairs. Les réseaux techniques facilitent là aussi la coordination du travail collaboratif et des échanges qui s'organisent sur un mode distribué. Les collaborateurs ont néanmoins 
éprouvé le besoin d'ajouter des règles garantissant la disponibilité juridique des contributions et gouvernant la production. L'utilisation de licences libres garantit la production collective contre toute appropriation privative ; et une auto-régulation par les pairs évite l'intégration d'œuvres préexistantes sur lesquelles des droits seraient encore réservés. Des exemples de créations en réseau très abouties sont fournis par les logiciels libres et par Wikipédia. Leur usage est régi par des licences libres. Selon ce modèle juridique, le contributeur devra apporter ses modifications selon les mêmes conditions de liberté que l'œuvre qu'il modifie, permettant la libre réutilisation et modification par tous tout en empêchant l'appropriation par certains. La participation à ces réseaux est également soumise à des règles très structurées, notamment sur Wikipédia, pouvant aller jusqu'à exclure les usagers ne les respectant pas. Ceci révèle une hiérarchie des contributeurs et éditeurs, certains bénéficiant de droits particuliers pour arbitrer les conflits qui restent néanmoins publics et débattus collectivement.

\section{Les réseaux de production par les pairs contrôlés par des entreprises}

Les réseaux de production collaborative de connaissances constituent un modèle économique qui a prouvé sa viabilité, adossé à une gouvernance politique et juridique structurée. Cependant, ce modèle de production collaborative est également utilisé au sein d'environnements commerciaux qui vont alors priver les pairs de la mise en commun du résultat de leur travail collectif. Ainsi, les données circulant sur les médias et réseaux sociaux participent à la création de valeur pour les entreprises qui développent et hébergent ces services, comme le montre par exemple l'introduction en bourse de Facebook, ou le rachat du site de blog Huffington Post par AOL, réalisés dans les deux cas au seul bénéfice de l'équipe organisatrice. Dans cette appropriation privée dite « du passager clandestin », ni les règles de gouvernance ni le produit de la connaissance n'appartiennent aux pairs qui perdent de surcroît le contrôle de leurs données personnelles.

Différents modèles de production collaborative par des pairs cohabitent donc sur les réseaux, et peuvent être catégorisés en fonction du mode de décision, centralisé ou distribué, des formes de création des règles d'organisation du réseau, et des conditions d'appropriation, privatives ou sur la base de biens communs (Fuster Morell, 2010).

Références bibliographiques

Aigrain, P., Cause commune : l'information entre bien commun et propriété, Paris, Fayard, coll. «Transversales », 2005.

Bodó, B., LAKATOS, Z., « Piracy Cultures : P2P and Cinematographic Movie Distribution in Hungary », International Journal of Communication, vol. 6, 2012.

BENKLER, Y., La richesse des réseaux, Marchés et libertés à l'heure du partage social, Lyon, Presses Universitaires de Lyon, 2009.

Fuster Morell, M., Governance of online Creation Communities: Provision of Infrastructure for the Building of Digital Commons, $\mathrm{PhD}$ thesis, European University Institute, 2010.

ShIRKY, C., Cognitive Surplus : Creativity and Generosity in a Connected Age, Penguin Press, 2010.

Ostrom, E., Governing the Commons : The Evolution of Institutions for Collective Action, Cambridge University Press, 1990.

Association Vecam (éd.), Libres Savoirs : Les biens communs de la connaissance. Produire collectivement, partager et diffuser les connaissances au XXI ${ }^{\mathrm{e}}$ siècle, Caen, C\&F Éditions, 2011. 